\title{
Dynamical efficiency of collisionless magnetized shocks in relativistic jets
}

\author{
Miguel A. Aloy and Petar Mimica \\ Departamento de Astronomía y Astrofísica, Universidad de Valencia, 46100, Burjassot, Spain
}

\begin{abstract}
The so-called internal shock model aims to explain the light-curves and spectra produced by non-thermal processes originated in the flow of blazars and gamma-ray bursts. A long standing question is whether the tenuous collisionless shocks, driven inside a relativistic flow, are efficient enough to explain the amount of energy observed as compared with the expected kinetic power of the outflow. In this work we study the dynamic efficiency of conversion of kinetic-tothermal/magnetic energy of internal shocks in relativistic magnetized outflows. We find that the collision between shells with a non-zero relative velocity can yield either two oppositely moving shocks (in the frame where the contact surface is at rest), or a reverse shock and a forward rarefaction. For moderately magnetized shocks (magnetization $\sigma \simeq 0.1$ ), the dynamic efficiency in a single two-shell interaction can be as large as $40 \%$. Hence, the dynamic efficiency of moderately magnetized shocks is larger than in the corresponding unmagnetized two-shell interaction. We find that the efficiency is only weakly dependent on the Lorentz factor of the shells and, thus internal shocks in the magnetized flow of blazars and gamma-ray bursts are approximately equally efficient.
\end{abstract}

Keywords: Hydrodynamics - (magnetohydrodynamics) MHD - Shock waves - gamma-rays: bursts - galaxies: BL Lacertae objects: general

PACS: $95.30 . \mathrm{Qd}$, 95.30.Sf, 98.54.Cm, 98.70.Rz, 47.40.-x, 47.40.Nm, 47.75.+f

\section{INTRODUCTION}

Internal shocks (ISs) [1] are invoked to explain the variability of blazars [e.g., 2, 3] and the light curves of the prompt phase of gamma-ray bursts (GRBs) [4, 5, 6]. A long standing concern in this model is the question whether it is efficient enough to explain the relation between the observed energies both in the prompt GRB phase and in the afterglow [see, e.g., 7, 8, 9, 10]. To assess the efficiency of the internal shock model, most of the previous works focus on the comparison between the observed light curves and the model predictions employing a simple inelastic collision of two-point masses $[7,11,12,13,14]$. Less attention has been paid to the hydrodynamic effects during the shell collision [but see, 9, 15, 16, 3, 17].

The ejecta in GRBs and blazars may be significantly magnetized, particularly if they originate from a Poynting-flux-dominated flow [18]. Forming shocks in highly magnetized media is challenging since the Alfvén speed approaches the speed of light. Therefore, to account for the observed phenomenology it is necessary to address how efficient can be shock dissipation of the internal collisions in arbitrarily magnetized flows. This question has been partly considered by a few recent works [e.g., 19, 20], and only very recently has been studied extensively [21].

The base for a study the efficiency of internal collisions is the determination of the dynamic efficiency of a single binary collision, i.e., the efficiency of converting the kinetic 
energy of the colliding fluid into thermal and/or magnetic energy. Thus, we model ISs as results of the collision of (magneto-)hydrodynamic shells of plasma with a non-zero relative velocity. The contact surface, where the interaction between the shells occurs, can break up either into two oppositely moving shocks (in the frame where the contact surface is at rest), or into a reverse shock and a forward rarefaction. The determination of whether one or the other possibility occurs is computed by estimating the invariant relative velocity between the fastest and the slowest shell, i.e., by solving the relativistic magnetohydrodynamic (RMHD) Riemann problem posed by the piecewise uniform states given by the physical quantities on the two interacting shells (Sect. 2). In Section 3 we define precisely the notion of dynamic efficiency, both for shocks and rarefactions. We perform a parametric study of the binary shell collision dynamic efficiency in Section 4. Finally, the discussion and conclusions are listed in Section 5.

\section{RMHD RIEMANN PROBLEM}

The interaction between parts of the outflow with varying properties can be modeled in terms of Riemann problems, i.e. relativistic magnetohydrodynamic initial-value problems with two constant states separated by a discontinuity. This approach allows us to use our models to sample very finely a large parameter space. The approach is also justified by the fact that the flow is cold and ultrarelativistic, so that its lateral expansion is negligible. Thus, a description of the interactions assuming planar symmetry suffices to compute the dynamic efficiency of such interactions.

In the following we use subscripts $L$ and $R$ to denote properties of the (faster) left and (slower) right state, respectively. We normalize the rest-mass density $\rho$ to $\rho_{R}$, the energy density to $\rho_{R} c^{2}$ ( $c$ is the speed of light) and the magnetic field strength to $c \sqrt{4 \pi \rho_{R}}$.

For the initial thermal pressure of both states we assume that it is a small fraction of the density, $p_{L}=\chi \rho_{L}$ and $p_{R}=\chi$, and that magnetic fields are perpendicular to the direction of the flow propagation. The remaining parameters determining the RMHD Riemann problem are: the density contrast $\rho_{L}$, the Lorentz factor of the right state $\Gamma_{R}$, the relative Lorentz factor difference $\Delta g:=\left(\Gamma_{L}-\Gamma_{R}\right) / \Gamma_{R}$, and the magnetizations of left and right states, $\sigma_{L}:=B_{L}^{2} /\left(\Gamma_{R}^{2}(1+\Delta g)^{2} \rho_{L}\right)$ and $\sigma_{R}:=B_{R}^{2} / \Gamma_{R}^{2}$, where $B_{L}$ and $B_{R}$ are the lab frame magnetic field strengths of left and right states, respectively. Furthermore, we define the total (thermal + magnetic) pressure, the total specific enthalpy and the total energy density, respectively, as

$$
\begin{aligned}
p^{*}:= & p+\frac{B^{2}}{2 \Gamma^{2}}=p+\frac{\sigma \rho}{2}, \\
h^{*} & :=1+\varepsilon+p / \rho+\sigma, \\
e^{*} & :=\rho(1+\varepsilon)+\frac{\sigma \rho}{2},
\end{aligned}
$$

where $\varepsilon$ denotes the specific internal energy.

The typical structure of the flow after the break-up of the initial discontinuity consists of the two initial states, and two intermediate states separated by a contact discontinuity (CD) -see [22]. The total pressure and velocity are the same on both sides of the CD. The quantity $\sigma / \rho$ is uniform everywhere, except across the $\mathrm{CD}$, where it can have a 
jump. We denote the total pressure of intermediate states $p_{S}^{*}$, and rest-mass density left and right of the $\mathrm{CD}$ as $\rho_{S, L}$ and $\rho_{S, R}{ }^{1}$.

One of the key steps in solving a Riemann problem is to determine under which conditions shocks can form. This happens when the Lorentz-invariant relative velocity between the left and right states, measured in the frame of the CD, $v_{l r}:=\left(v_{l}-v_{r}\right) /(1-$ $\left.v_{l} v_{r}\right)$ is larger than the limiting value [21]

$$
\left(v_{l r}\right)_{2 S}=\left\{\begin{array}{l}
\sqrt{\frac{\left(p_{L}^{*}-p_{R}^{*}\right)\left(e_{S, R}^{*}\left(p_{L}^{*}\right)-e_{R}^{*}\right)}{\left(e_{S, R}^{*}\left(p_{L} *\right)+p_{R}^{*}\right)\left(e_{R}^{*}+p_{L}^{*}\right)}} \text { if } p_{L}^{*}=p_{S}^{*}>p_{R}^{*} \\
\sqrt{\frac{\left(p_{R}^{*}-p_{L}^{*}\right)\left(e_{S, L}^{*}\left(p_{R}^{*}\right)-e_{L}^{*}\right)}{\left(e_{S, L}^{*}\left(p_{R}^{*}\right)+p_{L}^{*}\right)\left(e_{L}^{*}+p_{R}^{*}\right)}} \text { if } p_{L}^{*}<p_{R}^{*}=p_{S}^{*}
\end{array}\right.
$$

Generally, the quantity $\left(v_{l r}\right)_{2 S}$ can be only determined numerically. If $\left(v_{l r}\right)<\left(v_{l r}\right)_{2 S}$, a single shock and a rarefaction emerge from the initial discontinuity.

\section{ENERGY DISSIPATION EFFICIENCY OF ISS}

To study ISs we idealize interactions of parts of the outflow moving with different velocities as collisions of homogeneous shells. In our model the faster (left) shell catches up with the slower (right) one yielding, in some cases, a pair of shocks propagating in opposite directions (as seen from the CD frame). In order to cover a wide range of possible flow Lorentz factors and shell magnetizations, we assume that initially, the flux of energy in the lab frame is uniform and the same in both shells (see [21]). We then compute the break up of the initial discontinuity between both shells using the exact Riemann solver developed by Romero et al. [22], assuming an ideal gas equation of state $($ EoS $)$ with an adiabatic index $\hat{\gamma}=4 / 3$.

Efficiency of energy dissipation by a shock. To model the dynamic efficiency of energy dissipation we follow the approach described in [21]. By using the exact solver we determine the existence of shocks and (in case one or two shocks exist) obtain the hydrodynamic state of the shocked fluid. We use subscripts $S, L$ and $S, R$ to denote shocked portions of left and right shells, respectively. In the following we treat the efficiency of each shock separately.

Reverse shock. To compute the efficiency we need to compare the energy content of the initial (unshocked) faster shell with that of the shocked shell at the moment when RS has crossed the initial shell. Assuming an initial shell width $\Delta x$, we define total initial

\footnotetext{
${ }^{1}$ In the context of ISs, if the flow is ultrarelativistic in the direction of propagation, the velocity components perpendicular to the flow propagation should be negligibly small and, hence, they are set up to zero in our model. If such velocities were significant, appreciable changes in the Riemann structure may result as pointed out in Aloy and Rezzolla [23] or Aloy and Mimica [24].
} 
kinetic, thermal and magnetic energy [21]

$$
\begin{aligned}
E_{K}(\Gamma, \rho, \Delta x) & :=\Gamma(\Gamma-1) \rho \Delta x \\
E_{T}(\Gamma, \rho, p, \Delta x) & :=\left[(\rho \varepsilon+p) \Gamma^{2}-p\right] \Delta x \\
E_{M}(\Gamma, \rho, \sigma, \Delta x) & :=\left(\Gamma^{2}-1 / 2\right) \rho \sigma \Delta x .
\end{aligned}
$$

When the RS crosses the whole initial shell, the length of the compressed shell (i.e., the fluid between the RS and the CD) is $\zeta_{L} \Delta x$, where $\zeta_{L}:=\left(v_{C D}-v_{S, L}\right) /\left(v_{L}-v_{S, L}\right)<1$, and $v_{C D}$ and $v_{S, L}$ are velocities (in the lab frame) of the contact discontinuity and the RS, both obtained from the solver. We normalize the energies taking $\Delta x=1$, and define the dynamic thermal and magnetic efficiencies, i.e., the fraction of the initial energy that the RS has converted into thermal and magnetic energy, respectively, as

$$
\begin{aligned}
\varepsilon_{T, L} & :=\frac{E_{T}\left(\Gamma_{S, L}, \rho_{S, L}, p_{S, L}, \zeta_{L}\right)-E_{T}\left(\Gamma_{R}(1+\Delta g), \rho_{L}, \chi \rho_{L}, 1\right)}{E_{0}} \\
\varepsilon_{M, L} & :=\frac{E_{M}\left(\Gamma_{S, L}, \rho_{S, L}, \sigma_{S, L}, \zeta_{L}\right)-E_{M}\left(\Gamma_{R}(1+\Delta g), \rho_{L}, \sigma_{L}, 1\right)}{E_{0}}
\end{aligned}
$$

where $E_{0}$ is the total initial energy of both shells

$$
\begin{aligned}
E_{0}:= & E_{K}\left(\Gamma_{R}(1+\Delta g), \rho_{L}, 1\right)+E_{K}\left(\Gamma_{R}, 1,1\right)+E_{T}\left(\Gamma_{R}(1+\Delta g), \rho_{L}, \chi \rho_{L}, 1\right)+ \\
& E_{T}\left(\Gamma_{R}, 1, \chi, 1\right)+E_{M}\left(\Gamma_{R}(1+\Delta g), \rho_{L}, \sigma_{L}, 1\right)+E_{M}\left(\Gamma_{R}, 1, \sigma_{R}, 1\right) .
\end{aligned}
$$

Forward shock. In complete analogy, we define the thermal and magnetic efficiencies for the forward shock,

$$
\begin{aligned}
\varepsilon_{T, R} & :=\frac{E_{T}\left(\Gamma_{S, R}, \rho_{S, R}, p_{S, R}, \zeta_{R}\right)-E_{T}\left(\Gamma_{R}, 1, \chi, 1\right)}{E_{0}} \\
\varepsilon_{M, R} & :=\frac{E_{M}\left(\Gamma_{S, R}, \rho_{S, R}, \sigma_{S, R}, \zeta_{R}\right)-E_{M}\left(\Gamma_{R}, 1, \sigma_{R}, 1\right)}{E_{0}},
\end{aligned}
$$

where $\zeta_{R}:=\left(v_{S, R}-v_{C D}\right) /\left(v_{S, R}-v_{R}\right)<1$, and $v_{S, R}$ is the lab frame velocity of the FS.

Combining equations (6), (7), (9) and (10) we define the dynamic thermal and magnetic efficiency of ISs

$$
\varepsilon_{T}:=\varepsilon_{T, L}+\varepsilon_{T, R}, \quad \varepsilon_{M}:=\varepsilon_{M, L}+\varepsilon_{M, R}
$$

Efficiency of energy dissipation by a rarefaction. In a rarefaction there is a net conversion of magnetic and/or thermal energy into kinetic energy, thus the net dynamic efficiency produced by a rarefaction, defined as in e.g., Eq. (7), should be negative. Therefore, it is possible that in some cases the total (left plus right) thermal or magnetic efficiency (Eqs. (11)) becomes negative. However, this situation does not correctly model the fact that, in cases where a single shock exists, it is still able to radiate away part of the thermal or magnetic energy behind it. Thus, we set $\varepsilon_{T, L}=\varepsilon_{M, L}=0$ $\left(\varepsilon_{T, R}=\varepsilon_{M, R}=0\right)$ if the reverse (forward) shock is absent. 

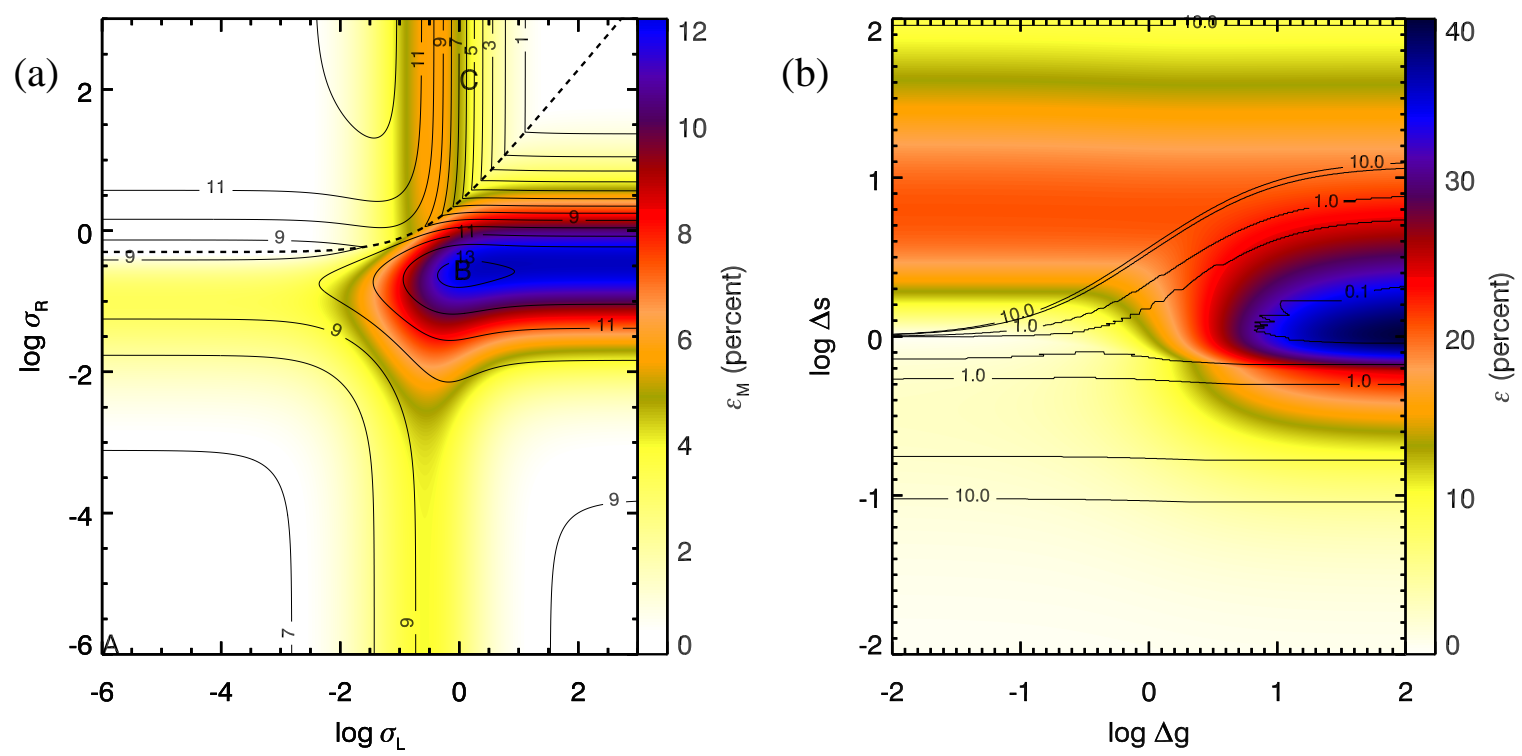

FIGURE 1. Panel (a): contours of the total dynamic efficiency $\varepsilon_{T}+\varepsilon_{M}$ (Eq. (11)) in the GRB regime $\left(\Gamma_{R}=100, \Delta g=1\right.$; left panel) for different combinations of $\left(\sigma_{L}, \sigma_{R}\right)$. Contours indicate the efficiency in percent and their levels are 1, 2, 3, 4, 5, 6, 7, 8, 9, 10, 11, 12 and 13. In the region of the parameter space above the dashed line there is no forward shock, while the reverse shock is always present for the considered parametrization. Colors: magnetic efficiency $\varepsilon_{M}$ in percent. Panel $(b)$ : The color scale indicates the value of the maximum total dynamic efficiency (in percent) as a function of the parameter pair $(\Delta g, \Delta s)$. The values of the rest of the parameters are fixed to $\Gamma_{R}=100$, and $\chi=10^{-4}$. Contours: magnetization of the slowest shell: $\sigma_{R}=0.1,0.5,1,5$ and 10 .

\section{PARAMETRIC STUDY OF THE DYNAMIC EFFICIENCY}

Next we study the dynamic dissipation efficiency in the process of collision of cold, magnetized shells. The shells are assumed to be cold because in the standard fireball model, almost all the internal energy of the ejecta has been converted to kinetic energy before ISs happen. If the ejecta were accelerated by magnetic fields, then the flow is cold (i.e., $\chi \ll 1$ ) all the way from the beginning to the internal shock phase. Thus, we fix $\chi=10^{-4} \ll 1$, to model initially cold shells. We set $\Delta g=1$ as a reference value, and consider the dependence of the total dynamical efficiency $\left(\varepsilon_{T}+\varepsilon_{M}\right)$ on the individual magnetization of each shell $\sigma_{L}$ and $\sigma_{R}$ Fig. 1(a). For the purpose of this study we take $\Gamma_{R}=100$, which is a typical value of GRB outflows. Smaller values, $\Gamma_{R}=10$, more representative of blazar outflows, do not yield significant differences in the total dynamical efficiency [21].

The maximum efficiency (where $\left.\varepsilon_{T}+\varepsilon_{M} \gtrsim 0.13\right)$ is attained for $\left(\sigma_{R}, \sigma_{L}\right) \approx(0.2,1)$. In the region above the dashed line of Fig. 1(a) the FS is absent and, thus, since only the RS dissipates the initial energy, the efficiency slightly drops. However, the transition between the regime where the two shocks operate or only the RS exists is smooth. The reason being that the efficiency below the separatrix of the two regimes and close to it is dominated by the contribution of RS. As expected, when either $\sigma_{L}$ or $\sigma_{R}$ approach low values, the dynamic efficiency ceases to depend on them.

We illustrate the differences in terms of dynamical efficiency by choosing three 
characteristic models in the parameter space (letters $A, B$ and $C$; Fig. 1a). Model $A$ corresponds to a prototype of interaction between non-magnetized shells $\left(\sigma_{L}=\sigma_{R}=\right.$ $\left.10^{-6}\right)$, model $B$ is located at the maximum efficiency $\left(\sigma_{L}=0.8, \sigma_{R}=0.2\right)$, and model $C$ corresponds to the case when the FS is absent $\left(\sigma_{L}=1, \sigma_{R}=10^{2}\right)$. All three models have a substantial dynamic efficiency, but there is a qualitative difference among them. In model $A$, ISs dissipate kinetic to thermal energy only (thermal efficiency). In model $B$ shocks mainly compress the magnetic field (magnetic efficiency) and dissipate only a minor fraction of the initial kinetic and magnetic energy to thermal energy. Finally, in the model $C$ only the RS is active.

Values $\Delta g<2$ between adjacent parts of the flow are motivated by the results of numerical simulations of relativistic outflows [e.g., 26, 27, 28, 29, 30, 31, 32, 33] (but see, e.g., [34], who find $\Delta g \sim 1-19$ appropriate to model Mrk 421). This adjacent flow regions can be approximated as pairs of shells whose binary collision we are considering here. However, it has been confirmed by several independent works (e.g., [7, 8, 9, 15]) that, in order to achieve a high efficiency (more than a few percent) in internal collisions of unmagnetized shells, the ratio between the maximum $\left(\Gamma_{\max }\right)$ and the minimum $\left(\Gamma_{\min }\right)$ Lorentz factor of the distribution of initial shells should be $\Gamma_{\max } / \Gamma_{\min }>10$.

In view of these results, we have also made an extensive analysis of the dependence of the dynamic efficiency on $\Delta g$. Since we are also interested in evaluating the influence of the magnetic fields on the results, we define a new variable $\Delta s=\left(1+\sigma_{L}\right) /\left(1+\sigma_{R}\right)$, and plot (Fig. 1b) the value of the maximum efficiency reached for every combination $(\Delta g, \Delta s)$ and fixed values of the rest of the parameters $\left(\Gamma_{R}=100, \chi=10^{-4}\right)$. We find that the maximum total dynamic efficiency grows (non-monotonically) with increasing $\Delta g$ (Fig. 1b), in agreement with the above mentioned works for unmagnetized shell collisions. Indeed, a large value $\Delta g \gtrsim 10$ yields dynamic efficiency values $\sim 40 \%$ if both shells are moderately magnetized $\left(\sigma_{R} \sim \sigma_{L} \lesssim 0.1\right)$. Nevertheless, the amount of increase of efficiency with $\Delta g$ depends strongly on $\Delta s$. For $|\Delta s| \gtrsim 1$, corresponding to cases where the slower shell is highly magnetized $\left(\sigma_{R} \gtrsim 4\right)$, the maximum dynamic efficiency is almost independent of $\Delta g$; while for $|\Delta s| \lesssim 1$, the maximum dynamic efficiency displays a strong, non-monotonic dependence on $\Delta g$.

It is remarkable that values $5 \lesssim \Delta s \lesssim 100$ yield dynamic efficiencies in excess of $\sim 20 \%$, regardless of the relative Lorentz factor between the two shells. In this region of the parameter space the maximal dynamic efficiency happens when $\sigma_{R}>10, \sigma_{L}>50$, and the total dynamic magnetic efficiency dominates the total dynamic efficiency.

\section{DISCUSSION}

In this work we estimate the dynamic efficiency of conversion of kinetic-tothermal/magnetic energy in collisions (ISs) of magnetized shells in relativistic outflows. A fundamental difference between the internal collisions in magnetized and unmagnetized outflows is the fact that in the former case not only shocks but also rarefactions can form. Thus, one would naturally expect a reduced dynamic efficiency in the magnetized case. However, we find that the dynamic efficiency reaches values $\sim 10 \%-40 \%$, in a wide range of the parameter space typical for relativistic outflows of astrophysical interest (blazars and GRBs). Thus, the dynamic efficiency of moderately magnetized 
shell interactions is larger than in the corresponding unmagnetized case. This is because when the shells are moderately magnetized, most of the initial shell kinetic energy is converted to magnetic energy, rather than to thermal energy.

The difference in efficiency between flows in blazars $\left(\Gamma_{R}=10\right)$ and GRBs $\left(\Gamma_{R}=100\right)$ is very small if $\Delta g$ is fixed. From theoretical and numerical grounds, values of $\Delta g \simeq 1$ seem reasonable, and $\Delta g=1$ has been taken as a typical value for both blazars and GRB jets, which brings maximum efficiency when the magnetizations of the colliding shells are $\left(\sigma_{L}, \sigma_{R}\right) \simeq(1,0.2)$. Larger dynamic efficiency values $\sim 40 \%$ are reached if $\Delta g \gtrsim 10$ and $|\Delta s| \lesssim 0.1$, corresponding to cases where the magnetization of both shells is moderate $\left(\sigma_{R} \simeq \sigma_{L} \lesssim 0.1\right)$.

In the limit of low magnetization of both shells, the kinetic energy is mostly converted into thermal energy, where the increased magnetic energy in the shocked plasma is only a minor contribution to the total dynamic efficiency, i.e., $\varepsilon_{T} \ll \varepsilon_{M}$. Here we find that as the magnetization of the shells grows, the roles of $\varepsilon_{T}$ and $\varepsilon_{M}$ are exchanged, so that $\varepsilon_{T}<\varepsilon_{M}$ (at the maximum dynamic efficiency $\varepsilon_{T} \simeq 0.1 \varepsilon_{M}$ ). If the magnetization of both shells is large, the dynamic efficiency decreases again because producing shocks in highly magnetized media is very difficult. All these conclusions are independent on the EoS used to model the plasma [21].

The comparison of our results with previous analytic or semi-analytic works is not straightforward, since, generally, they do not compute the (magneto-)hydrodynamic shell evolution, and they include multiple interactions of a number of dense shells. The bottom line in these previous works is that internal collisions of unmagnetized shells can be extremely efficient ( $\sim 100 \%$; [8]) if the spread of the Lorentz factor (i.e., the ratio between the Lorentz factor of the faster, $\Gamma_{\max }$, and of the slower $\Gamma_{\min }$ shell in the sample) is large $\left(\Gamma_{\max } / \Gamma_{\min }=10^{3}\right.$; e.g., [7, 35]). A more moderate spread of the Lorentz factor $\Gamma_{\max } / \Gamma_{\min }=10$, yields efficiencies $\sim 20 \%$. These high efficiencies result after a large number of binary collisions. Since more than a single collision is included, the kinetic energy remaining in the flow after the first generation of collisions, can be further converted into internal energy as subsequent generations of collisions take place. In contrast, we find that moderate magnetizations of both shells $(\sigma \lesssim 0.1)$ and $\Delta g \gtrsim 10$ (which would roughly correspond to $\Gamma_{\max } / \Gamma_{\min }=9$ ) are enough for a single binary collision to reach a total dynamic efficiency of $\sim 40 \%$.

We point out that the energy radiated in the collision of magnetized shells is only a fraction, $f_{r} \lesssim 1$ [e.g., 36, 37, 8] of the energy dynamically converted into thermal or magnetic energy.Hence, a single binary collision between moderately magnetized shells may yield radiative efficiencies $\sim 0.4 f_{r}$. Therefore, (1) binary collisions in relativistic magnetized flows are efficient enough, from the dynamical point of view, to be a valid mechanism to dissipate the bulk kinetic energy of relativistic ejecta, and (2) the main restriction on the radiative efficiency comes from the radiation mechanism setting the limiting factor $f_{r}$. The estimated dynamic efficiency in the binary collision of magnetized shells will be considered in a future work by computing the radiative efficiency using the method devised in [38]. 


\section{ACKNOWLEDGMENTS}

MAA is an Starting Independent Grant fellow of the European Research Councill. We acknowledge the support from the Spanish Ministry of Education and Science through grants AYA2007-67626-C03-01 and CSD2007-00050. The authors thankfully acknowledge the computer resources, technical expertise and assistance provided by the Barcelona Supercomputing Center - Centro Nacional de Supercomputación.

\section{REFERENCES}

1. M. J. Rees, and P. Meszaros, ApJL 430, L93-L96 (1994).

2. M. Spada, G. Ghisellini, D. Lazzati, and A. Celotti, MNRAS 325, 1559 (2001).

3. P. Mimica, M. A. Aloy, E. Müller, and W. Brinkmann, A\&A 441, 103-115 (2005).

4. R. Sari, and T. Piran, ApJL 455, L143 (1995).

5. R. Sari, and T. Piran, ApJ 485, 270 (1997).

6. F. Daigne, and R. Mochkovitch, MNRAS 296, 275-286 (1998).

7. S. Kobayashi, T. Piran, and R. Sari, ApJ 490, 92 (1997).

8. A. M. Beloborodov, ApJ 539, L25 (2000).

9. S. Kobayashi, and R. Sari, ApJ 542, 819 (2000).

10. Y. Fan, and T. Piran, MNRAS 369, 197 (2006).

11. D. Lazzati, G. Ghisellini, and A. Celotti, MNRAS 309, L13 (1999).

12. E. Nakar, and T. Piran, ApJ 572, L139 (2002).

13. C. Tanihata, T. Takahashi, J. Kataoka, and G. M. Madejski, ApJ 584, 153 (2003).

14. B. Zhang, and P. Mészáros, International Journal of Modern Physics A 19, 2385 (2004).

15. M. Kino, A. Mizuta, and S. Yamada, ApJ 611, 1021 (2004).

16. P. Mimica, M. A. Aloy, E. Müller, and W. Brinkmann, A\&A 418, 947-958 (2004).

17. Ž. Bošnjak, F. Daigne, and G. Dubus, $A \& A$ 498, 677 (2009).

18. V. V. Usov, Nature 357, 472-474 (1992).

19. Y. Z. Fan, D. M. Wei, and B. Zhang, MNRAS 354, 1031 (2004).

20. P. Mimica, M. A. Aloy, and E. Müller, $A \& A$ 466, 93-106 (2007).

21. P. Mimica, and M. A. Aloy, MNRAS 401, 525-532 (2010).

22. R. Romero, J. Martí, J. A. Pons, J. M. Ibáñez, and J. A. Miralles, JFM 544, 323-338 (2005).

23. M. A. Aloy, and L. Rezzolla, ApJL 640, L115-L118 (2006).

24. M. A. Aloy, and P. Mimica, ApJ 681, 84-95 (2008).

25. L. Rezzolla, and O. Zanotti, JFM 449, 395-411 (2001).

26. M. A. Aloy, E. Müller, J. M. Ibáñez, J. Martí, and A. MacFadyen, ApJL 531, L119-L122 (2000).

27. M. A. Aloy, H.-T. Janka, and E. Müller, $A \& A$ 436, 273-311 (2005).

28. A. Mizuta, T. Yamasaki, S. Nagataki, and S. Mineshige, ApJ 651, 960 (2006).

29. W. Zhang, S. E. Woosley, and A. I. MacFadyen, ApJ 586, 356 (2003).

30. W. Zhang, S. E. Woosley, and A. Heger, ApJ 608, 365 (2004).

31. B. J. Morsony, D. Lazzati, and M. C. Begelman, ApJ 665, 569 (2007).

32. D. Lazzati, B. J. Morsony, and M. C. Begelman, ApJL 700, L47 (2009).

33. A. Mizuta, and M. A. Aloy, ApJ 699, 1261 (2009).

34. M. Kino, and F. Takahara, MNRAS 383, 713 (2008).

35. S. Kobayashi, F. Ryde, and A. MacFadyen, ApJ 577, 302 (2002).

36. A. Panaitescu, M. Spada, and P. Mészáros, ApJ 522, L105 (1999).

37. P. Kumar, ApJ 523, L113 (1999).

38. P. Mimica, M.-A. Aloy, I. Agudo, J. M. Martí, J. L. Gómez, and J. A. Miralles, ApJ 696, 1142 (2009). 